\title{
DIRECT AND INVERSE SPECTRAL THEORY OF ONE-DIMENSIONAL SCHRÖDINGER OPERATORS WITH MEASURES
}

\author{
ALI BEN AMOR AND CHRISTIAN REMLING
}

\begin{abstract}
We present a direct and rather elementary method for defining and analyzing one-dimensional Schrödinger operators $H=-d^{2} / d x^{2}+\mu$ with measures as potentials. The basic idea is to let the (suitably interpreted) equation $-f^{\prime \prime}+\mu f=z f$ take center stage. We show that the basic results from direct and inverse spectral theory then carry over to Schrödinger operators with measures.
\end{abstract}

\section{INTRODUCTION}

In this paper we will discuss the direct and inverse spectral theory of generalized Schrödinger operators

$$
H=-\frac{d^{2}}{d x^{2}}+\mu
$$

with a signed Borel measure $\mu$ as the potential. In the case where $\mu$ is absolutely continuous, $d \mu(x)=V(x) d x$, the theory of course reduces to the theory of the usual Schrödinger operator $-d^{2} / d x^{2}+V(x)$. Operators of the form (1.1) have been used to model singular interactions, which are located on small sets. See, for example, $[1,2,3,7,8]$.

We will make essential use of the fact that the problem is one-dimensional. Namely, we will use a direct approach based on the solutions of the (suitably interpreted) equation $-f^{\prime \prime}+\mu f=z f$ instead of quadratic forms, which is the customary tool in the theory of Schrödinger operators with measures. As one advantage of this approach, we do not need semi-boundedness assumptions on $\mu$; the drawback is that our method only works in the one-dimensional case.

We will discuss the general direct and inverse spectral theory of the operators $-d^{2} / d x^{2}+\mu$, using this approach. The setup and basic definitions may be found in the following section. Given this material, it will then be rather straightforward to extend the classical theory of the spectral representation of one-dimensional Schrödinger operators (based on the Titchmarsh-Weyl $m$ function). Therefore, our discussion of the direct spectral theory in Sect. 3 will be rather sketchy. However, things get more challenging in inverse spectral theory. Here, the smoothness of the potential plays a decisive role, and clearly, a measure $\mu$ can be even more singular

Date: September 4, 2003.

Key words and phrases. Schrödinger operator, spectral measure.

2000 Mathematics Subject Classification. Primary 34L15 34L40; secondary 34L16.

Ben Amor's work was supported by the Deutsche Forschungsgemeinschaft.

Remling's work was supported by the Heisenberg program of the Deutsche Forschungsgemeinschaft. 
than a potential. We will take the recent treatment of [9] as a guideline. More specifically, we will discuss and use the tool of de Branges spaces, and we will introduce a so-called $\phi$ function as the spectral data. Then, the inverse problem is to recover $\mu$ from $\phi$. It turns out, as expected, that $\phi$ has less smoothness than in the case where $\mu$ is absolutely continuous. Namely, $\phi$ is no longer absolutely continuous but (in general) only of bounded variation. This larger set of $\phi$ functions is in oneto-one correspondence to the class of (signed) measures $\mu$. These results will be discussed in Sect. 4-6.

\section{SCHRÖDINGER OPERATORS WITH MEASURES}

Let $\mu$ be a signed measure on the Borel subsets of $[0, \infty)$ with $|\mu|([0, N])<\infty$ for all $N>0$. We will define the operator $-d^{2} / d x^{2}+\mu$ as a self-adjoint operator on the Hilbert space $L_{2}(0, \infty)$ using a direct method rather than quadratic forms.

For $0 \leq a<b<\infty$, denote by $A C(a, b)$ the space of complex valued absolutely continuous functions on $(a, b)$. So $f \in A C(a, b)$ means there exists $g \in L_{1}(a, b)$ so that $f(x)=f(c)+\int_{c}^{x} g(t) d t$ for all $x \in(a, b)$ and with $c \in(a, b) . A C(0, \infty)$ is defined locally, that is, $f \in A C(0, \infty)$ if $f \in A C(0, b)$ for all $b>0$.

Fix $a>0$ and define, for $f \in A C(0, \infty)$,

$$
(A f)(x)=f^{\prime}(x)-\int_{a}^{x} f(t) d \mu(t)
$$

here, $\int_{a}^{x} \cdots$ is short-hand for

$$
\int_{a}^{x} f(t) d \mu(t)=\left\{\begin{array}{ll}
\int_{[a, x]} f(t) d \mu(t) & x \geq a \\
-\int_{(x, a)} f(t) d \mu(t) & x<a
\end{array} .\right.
$$

Clearly, $A f$ is only defined as an element of $L_{1, l o c}(0, \infty)$, or, in other words, almost everywhere with respect to Lebesgue measure. Af will play the role of a quasiderivative of $f$. We can now define the maximal operator associated with the differential expression $-d^{2} / d x^{2}+\mu$. Let

$$
\begin{aligned}
D(T) & =\left\{f \in L_{2}(0, \infty): f, A f \in A C(0, \infty),(A f)^{\prime} \in L_{2}(0, \infty)\right\} \\
T f & =-(A f)^{\prime} .
\end{aligned}
$$

It is clear that neither the domain of $T, D(T)$, nor the definition of $T$ depend on the choice of $a>0$. We want to establish regularity at $x=0$; in particular, we can then take $a=0$ in the above definitions.

Proposition 2.1. If $f \in D(T)$, then $f, A f \in A C[0, \infty)$.

Here, we say that $f \in A C[0, \infty)$ if there is a function $g \in L_{1, l o c}([0, \infty))$, so that $f(x)=f(0)+\int_{0}^{x} g(t) d t$. Put more precisely, Proposition 2.1 then claims that the functions $f, A f$ have extensions to $[0, \infty)$ which are in $A C[0, \infty)$.

Proof. To prove the statement on $A f$, we only need to observe that $(A f)^{\prime} \in$ $L_{2}(0, \infty) \subset L_{1, l o c}([0, \infty))$ and let $c \rightarrow 0+$ in

$$
(A f)(x)=(A f)(c)+\int_{c}^{x}(A f)^{\prime}(t) d t
$$

As for $f$, we have that

$$
f(x)=f(c)+\int_{c}^{x} f^{\prime}(t) d t=f(c)+\int_{c}^{x}(A f)(t) d t+\int_{c}^{x} d t \int_{c}^{t} d \mu(s) f(s) .
$$


By using Fubini's Theorem in the double integral, we thus see that

$$
\|f\|_{L_{\infty}(\epsilon, c)} \leq C+\|f\|_{L_{\infty}(\epsilon, c)} \int_{(\epsilon, c)}(s-\epsilon) d|\mu|(s) .
$$

The constant $C$ is independent of $\epsilon$, so by letting $\epsilon \rightarrow 0+$ and then taking $c>0$ sufficiently small, we see that $f$ is bounded on $(0, c)$. Hence $f^{\prime}(x)=(A f)(x)+$ $\int_{c}^{x} f(t) d \mu(t)$ is in $L_{1, l o c}([0, \infty))$.

This argument was local, so it is also true that $f, A f \in A C[0, N)$ if $\varphi f \in D(T)$ for some function $\varphi$ which is equal to 1 on $(0, N)$.

Proposition 2.1 allows us to take $a=0$ in the definition of $A f$. So from now on, we will define $A f$ for $f \in A C[0, N)$ by

$$
(A f)(x)=f^{\prime}(x)-\int_{[0, x]} f(t) d \mu(t) .
$$

To develop the theory of the self-adjoint realizations of $-d^{2} / d x^{2}+\mu$ along the usual lines (see for example [10]), we need Green's identity. Fix $N>0$ and let

$$
\begin{aligned}
D\left(T_{N}\right) & =\left\{f \in L_{2}(0, N): f, A f \in A C(0, N),(A f)^{\prime} \in L_{2}(0, N)\right\}, \\
T_{N} f & =-(A f)^{\prime} .
\end{aligned}
$$

By Proposition 2.1 and the remark following its proof (and also an analogous version for $x=N$ instead of $x=0$ ), we automatically have that $f, A f \in A C[0, N]$ for $f \in D\left(T_{N}\right)$. For $f \in D\left(T_{N}\right)$, we also fix a particular representative of $f^{\prime} \in L_{1}(0, N)$ by

$$
f^{\prime}(x):=(A f)(x)+\int_{[0, x]} f(t) d \mu(t) \quad(0 \leq x \leq N) ;
$$

here, we take the unique continuous representative of $A f$.

Theorem 2.2 (Green's identity). Suppose that $f, g \in D\left(T_{N}\right)$. Then

$$
\left\langle f, T_{N} g\right\rangle-\left\langle T_{N} f, g\right\rangle=\left.\left(\overline{f^{\prime}(x)} g(x)-\overline{f(x)} g^{\prime}(x)\right)\right|_{x=0} ^{x=N} .
$$

Proof. Integration by parts shows that

$$
\begin{aligned}
\left\langle f, T_{N} g\right\rangle-\left\langle T_{N} f, g\right\rangle=(\overline{(A f)(x)} g(x) & -\overline{f(x)}(A g)(x))\left.\right|_{x=0} ^{x=N} \\
& +\int_{0}^{N}\left(\overline{f^{\prime}(x)}(A g)(x)-\overline{(A f)(x)} g^{\prime}(x)\right) d x .
\end{aligned}
$$

In the integral, we plug in $A f, A g$ from (2.1) and use Fubini's theorem:

$$
\begin{aligned}
\int_{0}^{N}\left(\overline{f^{\prime}}(A g)-\overline{(A f)} g^{\prime}\right) d x & =\int_{0}^{N} d x \int_{[0, x]} d \mu(t)\left(\overline{f(t)} g^{\prime}(x)-\overline{f^{\prime}(x)} g(t)\right) \\
& =\int_{[0, N]} d \mu(t) \int_{t}^{N} d x\left(\overline{f(t)} g^{\prime}(x)-\overline{f^{\prime}(x)} g(t)\right) \\
& =g(N) \int_{[0, N]} \overline{f(t)} d \mu(t)-\overline{f(N)} \int_{[0, N]} g(t) d \mu(t)
\end{aligned}
$$

Since $\overline{(A f)(0)} g(0)-\overline{f(0)}(A g)(0)=\overline{f^{\prime}(0)} g(0)-\overline{f(0)} g^{\prime}(0)$, the asserted identity now follows. 
The theory of self-adjoint extensions depends on Green's identity, but not on the particular form of the differential expression itself, so we have the same theory here as in the classical case (where $d \mu(x)=V(x) d x$ ). For a detailed exposition of this theory, see [10]. $T_{N}$ is self-adjoint on a domain $D \subset D\left(T_{N}\right)$ if $D\left(T_{N}\right) / D$ is two-dimensional and the Lagrange form $L(f, g)=\left.\left(\overline{f^{\prime}(x)} g(x)-\overline{f(x)} g^{\prime}(x)\right)\right|_{x=0} ^{x=N}$ vanishes on $D$. These domains $D$ can be described in terms of boundary conditions. Here, we are interested in separated boundary conditions, that is, we require that the contributions from $x=0$ and $x=N$ vanish separately on $D$. An elementary argument shows that then the admissable boundary conditions are precisely given by

$$
f(0) \cos \alpha+f^{\prime}(0) \sin \alpha=0, \quad f(N) \cos \beta+f^{\prime}(N) \sin \beta=0,
$$

with $\alpha, \beta \in[0, \pi)$. Here, $f(0)$ and $f(N)$ are well defined because $f \in A C[0, N]$, and $f^{\prime}(0)$ and $f^{\prime}(N)$ are determined from $(2.2)$.

Now observe that the operators just defined are independent of $\mu(\{0\})$. Indeed, if $f \in D\left(T_{N}\right)$, the values of $f(0)$ and $f^{\prime}(0)$ do not change if we vary $\mu(\{0\})$. However, this does not necessarily mean that one should dismiss $\mu(\{0\})$ and view $\mu$ as a measure on $(0, N)$. Rather, we can make good use of $\mu(\{0\})$ in the following way: By the observation made at the end of the proof of Theorem 2.2, the boundary conditions at $x=0$ may also be written in the form $f(0) \cos \gamma+(A f)(0) \sin \gamma=0$, where $\gamma$ varies over $[0, \pi)$. Let us fix the boundary condition $(A f)(0)=0$. Since $(A f)(0)=f^{\prime}(0)-\mu(\{0\}) f(0)$, we now get all boundary conditions except Dirichlet boundary conditions $(f(0)=0)$ by adjusting $\mu(\{0\})$. Dirichlet boundary conditions play a special role for other reasons as well. So from now on, we work with the boundary conditions

$$
(A f)(0)=0, \quad f(N) \cos \beta+f^{\prime}(N) \sin \beta=0 .
$$

Of course, this is only a minor detail, but we will find it very convenient to use (2.4) and vary $\mu(\{0\})$ rather than use (2.3) and vary $\alpha(\alpha \neq 0)$.

Next, we study the solutions of the equation $-f^{\prime \prime}+\mu f=z f+g$. Clearly, this needs to be interpreted properly. The following definition suggests itself.

Definition 2.1. Let $g \in L_{1}(0, N), z \in \mathbb{C}$. We say that a function $f:(0, N) \rightarrow \mathbb{C}$ solves the equation

$$
-f^{\prime \prime}+\mu f=z f+g
$$

on $(0, N)$ if $f, A f \in A C(0, N)$ and $-(A f)^{\prime}=z f+g$ almost everywhere (with respect to Lebesgue measure) on $(0, N)$.

We have the usual existence and uniqueness results for the initial value problems associated with (2.5).

Theorem 2.3. Let $g \in L_{1}(0, N), z \in \mathbb{C}$ and $c, c^{\prime} \in \mathbb{C}$. Then the equation (2.5) has a unique continuous solution on $[0, N]$ such that $f(0)=c,(A f)(0)=c^{\prime}$. Moreover, $f(x, z)$ is entire in $z$ for every fixed $x \in[0, N]$.

Proposition 2.1 and the remark following its proof show that if $f$ solves (2.5) on $(0, N)$, then in fact $f, A f \in A C[0, N]$.

Sketch of proof. The proof is essentially the same as the one for differential equations (see, e.g., [4, Chapter 1]), so we only sketch the proof. 
By integrating twice and using Fubini's theorem, we see that (2.5) together with the initial conditions $f(0)=c,(A f)(0)=c^{\prime}$ is equivalent to the integral equation

$$
f(x)=c+c^{\prime} x+\int_{[0, x]}(x-t) f(t)(d \mu(t)-z d t)-\int_{0}^{x}(x-t) g(t) d t .
$$

Here, we seek continuous solutions $f$. If such an $f$ solves (2.6), then $f, A f$ are automatically absolutely continuous.

The right-hand side of (2.6) defines a contractive mapping on $C[0, \eta]$, provided $\eta>0$ is chosen sufficiently small. This yields existence and uniqueness on the interval $[0, \eta]$, and this argument may be repeated (with $\eta$ independent of the left endpoint) to obtain existence and uniqueness on all of $[0, N]$. Holomorphic dependence on $z$ also follows from this method.

We believe that the above interpretation of $-f^{\prime \prime}+\mu f=z f+g$ is natural; this is also confirmed by the following fact.

Theorem 2.4. If the function $f$ solves (2.5) in the sense of Definition 2.1 on $(0, N)$, then

$$
-f^{\prime \prime}+f \mu=z f+g
$$

in the sense of distributions on $(0, N)$.

Proof. We must show that the distributional derivative of the function $F(x):=$ $\int_{[0, x]} f(t) d \mu(t)$ is equal to the measure $F^{\prime}=f \mu$. Indeed, it follows from this fact that $(A f)^{\prime}=f^{\prime \prime}-f \mu$ (distributional derivatives), so the claim holds.

Let $\varphi \in C_{0}^{\infty}(0, N)$ be a test function. By the definition of the distributional derivative,

$$
\begin{aligned}
\left(F^{\prime}, \varphi\right) & =-\left(F, \varphi^{\prime}\right)=-\int_{0}^{N} d x \varphi^{\prime}(x) \int_{[0, x]} f(t) d \mu(t) \\
& =-\int_{[0, N]} f(t) d \mu(t) \int_{t}^{N} \varphi^{\prime}(x) d x=\int_{(0, N)} \varphi(t) f(t) d \mu(t)=(f \mu, \varphi),
\end{aligned}
$$

as desired.

We also see from the preceding result and its proof that $f^{\prime \prime}=(A f)^{\prime}+f \mu$ is a measure if $f$ solves an equation of the form (2.5). In particular, this is the case for $f \in D(T)$ or $f \in D\left(T_{N}\right)$. So, $f^{\prime}$ is then of bounded variation, with jumps precisely at the discrete points of the measure $f \mu$. The one-sided limits $f^{\prime}(x \pm):=\lim _{t \rightarrow x \pm} f^{\prime}(t)$ exist everywhere. The choice (2.2) (which we also use if $f$ solves (2.5)) implies that $f^{\prime}(x+)=f^{\prime}(x)$, and thus $f^{\prime}(x)-f^{\prime}(x-)=f(x) \mu(\{x\})$.

With this notation, we also have that $(A f)(0)=f^{\prime}(0-)$. This remark throws additional light on our discussion above of boundary conditions at $x=0$.

If $f, g$ are solutions of the homogenous equation $-y^{\prime \prime}+\mu y=z y$, then their Wronskian is defined as $W(f, g)=f(x) g^{\prime}(x)-f^{\prime}(x) g(x)$.

Proposition 2.5. The Wronskian of two solutions of the same equation is independent of $x$. 
Proof. This follows from the following computation:

$$
\begin{aligned}
& 0= \int_{0}^{N}\left(f(x)(A g)^{\prime}(x)-(A f)^{\prime}(x) g(x)\right) d x \\
&=\left.(f(x)(A g)(x)-(A f)(x) g(x))\right|_{x=0} ^{x=N}+\int_{0}^{N}\left((A f)(x) g^{\prime}(x)-f^{\prime}(x)(A g)(x)\right) d x \\
&= W(N)-W(0)-f(N) \int_{[0, N]} g(t) d \mu(t)+g(N) \int_{[0, N]} f(t) d \mu(t) \\
& \quad+\int_{0}^{N} d x \int_{[0, x]} d \mu(t)\left(f^{\prime}(x) g(t)-f(t) g^{\prime}(x)\right) \\
&=W(N)-W(0)-f(N) \int_{[0, N]} g(t) d \mu(t)+g(N) \int_{[0, N]} f(t) d \mu(t) \\
& \quad+\int_{[0, N]} d \mu(t) \int_{t}^{N} d x\left(f^{\prime}(x) g(t)-f(t) g^{\prime}(x)\right) \\
&=W(N)-W(0) \quad
\end{aligned}
$$

Finally, we introduce the notation $u(x, z)$ for the solution $u$ of $-u^{\prime \prime}+\mu u=z u$ with the initial values $u(0)=1,(A u)(0)=0$. Note that $u$ satisfies the boundary condition at $x=0$. This solution $u$ will become important in the following sections.

\section{Spectral Representation}

Given the material from the preceding section, the theory of the spectral representation of the operators associated with $-d^{2} / d x^{2}+\mu$ can be developed as in the classical case where $\mu$ is absolutely continuous. We will therefore just compile some basic results and say relatively little about the proofs, which are completely analogous to the classical ones (see $[4,10]$ ).

The operators on $L_{2}(0, N)$ have purely discrete spectrum. This follows from the fact that the resolvent is a compact operator, and this, in turn, follows from an explicit formula for the integral kernel of the resolvent in terms of the solutions of $-u^{\prime \prime}+\mu u=z u$.

A spectral representation is thus obtained by expanding in eigenfunctions. Since these eigenfunctions must be multiples of the solutions $u(x, z)$ introduced at the end of Sect. 2, we can proceed as follows. Define

$$
(U f)(E)=\int u(x, E) f(x) d x .
$$

Then, by the above remarks, $U$ maps the original Hilbert space $L_{2}(0, N)$ unitarily onto $L_{2}\left(\mathbb{R}, d \rho_{N}^{(\beta)}\right)$, where

$$
\rho_{N}^{(\beta)}=\sum \frac{\delta_{E}}{\|u(\cdot, E)\|^{2}} .
$$

The sum is over the eigenvalues $E$ (which depend on $N$ and the boundary condition $\beta$ at $x=N)$ and $\delta_{E}$ denotes the Dirac measure at $E$, so $\delta_{E}(\{E\})=1, \delta_{E}(\mathbb{R} \backslash\{E\})=$ 0 . The denominator is necessary because the $u$ 's are not normalized.

For operators on the half-axis $(0, \infty)$, the situation is considerably more complicated, because the spectrum need not be discrete. However, as already explained, 
one may proceed exactly as in the case of a potential $(d \mu(x)=V(x) d x)$. There is the distinction between the limit point and limit circle case at infinity. In the latter case, one needs a boundary condition at infinity to obtain self-adjoint operators. In either case, one can define a Titchmarsh-Weyl $m$ function in the usual way. The measure $\rho$ from the Herglotz representation of this $m$ function is a spectral measure in the sense that $U$ from $(3.1)$ still maps $L_{2}(0, \infty)$ unitarily onto $L_{2}(\mathbb{R}, d \rho)$. Moreover, the transformed operator $U H U^{*}$ is multiplication by the variable in $L_{2}(\mathbb{R}, d \rho)$, but this will be less important for us.

It is, of course, no coincidence that these well known methods carry over to Schrödinger operators with measures. Rather, the treatment of [4, Chapter 9] does not depend on the precise form of the underlying differential equation, but only on Green's identity (see Theorem 2.2).

\section{DE BRAnges SPACES}

In this section, we want to follow [9] and use de Branges spaces as a tool in the spectral analysis of Schrödinger operators. For the definition and the properties of de Branges functions and spaces we refer the reader to $[5,9]$.

We fix $N>0$ and, as in [9], set $E_{N}(z):=u(N, z)+i u^{\prime}(N, z)$. Here, $u$ still is the solution defined at the end of Sect. 2 .

Proposition 4.1. The function $E_{N}$ is a de Branges function.

Proof. It is clear that $E_{N}$ is entire, so it remains to show that $\left|E_{N}(z)\right|>\left|E_{N}(\bar{z})\right|$ for all $z \in \mathbb{C}^{+}=\{z \in \mathbb{C}: \operatorname{Im} z>0\}$. Let $\zeta, z \in \mathbb{C}$. Green's identity (Theorem 2.2), applied with $f=u(\cdot, z), g=u(\cdot, \zeta)$ gives

$$
\frac{\overline{u(N, z)} u^{\prime}(N, \zeta)-\overline{u^{\prime}(N, z)} u(N, \zeta)}{\bar{z}-\zeta}=\int_{0}^{N} \overline{u(N, z)} u(x, \zeta) d x .
$$

From the integral equation satisfied by $u(x, z)$ and the uniqueness of the solution we get $\overline{u(x, z)}=u(x, \bar{z})$. Therefore the left-hand side of (4.1) is equal to

$$
\frac{\overline{E_{N}(z)} E_{N}(\zeta)-E_{N}(\bar{z}) \overline{E_{N}(\bar{\zeta})}}{2 i(\bar{z}-\zeta)} .
$$

Hence for $z=\zeta \in \mathbb{C}^{+}$we have that $\left|E_{N}(z)\right|>\left|E_{N}(\bar{z})\right|$, as required.

Given a de Branges function $E$, one can form the de Branges space $B(E)$ based on $E$. One possible definition is given by

$$
B(E)=\left\{F: \mathbb{C} \rightarrow \mathbb{C}: F \text { entire, } F / E, F^{\#} / E \in H_{2}\right\}
$$

Here, $F^{\#}(z)=\overline{F(\bar{z})}$, and $H_{2}$ is the Hardy space on the upper half plane, that is, $f \in H_{2}$ precisely if $f$ is (defined and) holomorphic on the upper half plane and $\sup _{y>0} \int_{-\infty}^{\infty}|f(x+i y)|^{2} d x<\infty . B(E)$ is a Hilbert space with the scalar product $[F, G]=(1 / \pi) \int_{\mathbb{R}} \bar{F} G /|E|^{2} d \lambda$. These spaces $B(E)$ may be used in the spectral representation of Schrödinger operators. We will write $B\left(E_{N}\right)=: S_{N}(\mathrm{~S}$ for Schrödinger). Set

$$
V: S_{N} \longrightarrow L_{2}\left(\mathbb{R}, d \rho_{N}^{(\beta)}\right), V F=\left.F\right|_{\mathbb{R}}
$$

By using the arguments from [9, Sect. 3], we conclude that $V$ is unitary. So we may replace the space $L_{2}\left(\mathbb{R}, d \rho_{N}^{(\beta)}\right)$ in the spectral representation by the de Branges 
space $S_{N}$. We get an induced unitary map $U: L_{2}(0, N) \rightarrow S_{N}$ (which we still denote by $U$ ). It is given by

$$
(U f)(z)=\int u(x, z) f(x) d x .
$$

In particular,

$$
S_{N}=\left\{F(z)=\int_{0}^{N} u(x, z) f(x) d x: f \in L_{2}(0, N)\right\} .
$$

Finally, de Branges spaces have reproducing kernels $J_{z}$ (so $\left[J_{z}, F\right]=F(z)$ for all $F \in B(E))$. In the case of $S_{N}$, they are given by

$$
J_{z}(\zeta)=\int_{0}^{N} \overline{u(x, z)} u(x, \zeta) d x .
$$

The function $u^{\prime}(x, z)$ has jumps at the discrete points of $u(x, z) d \mu(x)$. More precisely, as observed in Sect. 2, we have that $u^{\prime}(x, z)-u^{\prime}(x-, z)=u(x, z) \mu(\{x\})$. So it would have been equally natural to work with $\widetilde{E}_{N}(z)=u(N, z)+i u^{\prime}(N-, z)$. However, this new de Branges function leads to the same de Branges space: $B\left(E_{N}\right)=$ $B\left(\widetilde{E}_{N}\right)$. This follows at once from [9, Theorem 7.2] because (using the notation from that reference) $\widetilde{A}(z)=A(z), \widetilde{B}(z)=B(z)-\mu(\{N\}) A(z)$. It is also quite clear form another point of view because replacing $u^{\prime}(N, z)$ by $u^{\prime}(N-, z)$ amounts to a change of boundary conditions at $x=N$, but the de Branges space $S_{N}$ is independent of this boundary condition.

As in [9], we will now describe the spaces $S_{N}$ in more detail. We prepare for this with two lemmas which describe the large $z$ asymptotics of $u(N, z)$ and $u^{\prime}(N, z)$.

Lemma 4.2. Write $z=k^{2}$. Then u satisfies the integral equation

$$
u(x, z)=\cos k x+\frac{1}{k} \int_{[0, x]} u(t, z) \sin k(x-t) d \mu(t) .
$$

Note that only even functions of $k$ occur, so there is no problem with the fact that there are two solutions to $z=k^{2}$ if $z \neq 0$. Moreover, these functions are also entire, so everything makes sense for $z=k=0$ as well.

Proof. Fix $z=k^{2} \neq 0$. For $k=0$, the following calculation also works but it must be slightly modified. From the equation statisfied by $u$ we get

$$
-\int_{0}^{x}(A u)^{\prime}(t) \sin k(x-t) d t=k^{2} \int_{0}^{x} u(t, z) \sin k(x-t) d t .
$$

Integrating the first term by parts and using Fubini's theorem, we get

$$
\begin{array}{r}
-k u(x)+k \cos k x+k^{2} \int_{0}^{x} u(t) \sin k(x-t) d t+ \\
\int_{[0, x]} u(t) \sin k(x-t) d \mu(t) \\
=k^{2} \int_{0}^{x} u(t) \sin k(x-t) d t
\end{array}
$$

which is the desired equation 
Lemma 4.3. Write $z=k^{2}$. Then, for large $|k|$, we have that

$$
\begin{aligned}
|u(x, z)-\cos k x| & \leq \frac{C_{N}}{|k|} \exp (|\operatorname{Im} k| x), \\
\left|u^{\prime}(x, z)+(1 / k) \sin k x\right| & \leq C_{N} \exp (|\operatorname{Im} k| x) .
\end{aligned}
$$

These estimates hold uniformly in $x \in[0, N]$.

Proof. Write $k=r+i t$ and let $f(x):=e^{-|t| x} u(x, z)$. Then from Lemma 4.2, we get

$$
M \equiv \sup _{x \in[0, N]}|f(x)| \leq 1+\frac{1}{|k|}|\mu|([0, N]) M .
$$

Hence $M \leq 2$, say, for large enough $k$. By using this estimate in (4.2) again, we get the desired estimate, with $C_{N}=2|\mu|([0, N])$

To prove the estimate on $u^{\prime}$, note that Lemma 4.2 implies that

$$
u^{\prime}(x, z)=-k \sin k x-\int_{[0, x]} u(t, z) \cos k(x-t) d \mu(t) .
$$

Indeed, the right-hand side is the distributional derivative of the right-hand side of (4.2) and it is also the correct representative because it is right continuous. The asserted estimate on $u^{\prime}$ now follows immediately.

Theorem 4.4. As a set, the space $S_{N}$ is given by

$$
S_{N}=\left\{F(z)=\int_{0}^{N} f(t) \cos \sqrt{z} t d t: f \in L_{2}(0, N)\right\} .
$$

This is the generalization of [9, Theorem 4.1], and, given Lemma 4.3, it has the same proof.

\section{The DIRECT SPECTRAL THEOREM}

It remains to analyze the possible scalar products on the de Branges spaces coming from generalized Schrödinger equations. To this end, we introduce a function $\phi$ that describes these scalar products and may thus be thought of as representing the spectral data.

We need some notation. We write $B V(-2 N, 2 N)$ for the set of real valued functions on $(-2 N, 2 N)$ that are of bounded variation. Given an even function $\phi \in B V(-2 N, 2 N)$, we set

$$
K(s, t):=\frac{1}{2}(\phi(s-t)+\phi(s+t)) .
$$

Define the integral operator $\mathcal{K}_{\phi}$ on $L_{2}(0, N)$ by

$$
\left(\mathcal{K}_{\phi} f\right)(t):=\int_{0}^{N} K(t, s) f(s) d s .
$$

Then $\mathcal{K}_{\phi}$ is a self-adjoint Hilbert-Schmidt operator. For the proof of the direct spectral theorem we will need an asymptotic formula for the Titchmarsh-Weyl $m$ function. We consider the problem on the half line $(0, \infty)$ with the modified measure $\mu_{N}=\chi_{[0, N]} \mu$. The $m$ function of this problem (with boundary condition $(A u)(0)=0)$ will be denoted by $m_{N}$, and $m_{0}$ will be the $m$ function for $\mu=0$, that is, $m_{0}(z)=(-z)^{1 / 2}$, where the square root is determined by the requirement that 
$\operatorname{Im} m(z)>0$ for $z \in \mathbb{C}^{+}$. If $z=k^{2}$ with $\operatorname{Im} k>0$ and $f$ is the solution of $-f^{\prime \prime}+$ $\mu_{N} f=k^{2} f$ with $f(x, k)=e^{i k x}$ for $x>N$, then $m_{N}\left(k^{2}\right)=-f(0, k) /(A f)(0, k)$.

Finally, we introduce $M_{N}(k)=m_{N}\left(k^{2}\right)$. The functions $m_{N}, m_{0}$ (originally defined on $\left.\mathbb{C}^{+}\right)$may be holomorphically continued to $\mathbb{C} \backslash(-\infty, 0]$, and $M_{N}$ extends to a meromorphic function on $\mathbb{C}$. In the following lemma, we work with these extensions. We refer the reader to [9] for a more careful discussion of these issues.

Lemma 5.1. a) The limit $\lim _{k \rightarrow 0} k M_{N}(k)$ exists.

b) For $\operatorname{Im} k \geq 0, k \notin(-\infty, 0]$, we have

$$
m_{N}\left(k^{2}\right)-m_{0}\left(k^{2}\right)=\frac{1}{k^{2}} \int_{[0, N]} e^{2 i k x} d \mu(x)+O\left(|k|^{-3}\right) .
$$

Proof. Part a) is proved as in [9]. We use the constancy of the Wronskian established in Proposition 2.5.

To prove part b), we also proceed as in the proof of [9, Lemma 4.3]. We claim that $g(x, k) \equiv f(x, k) e^{-i k x}$ is the unique solution of the integral equation

$$
g(x, k)=1+\frac{1}{2 i k} \int_{(x, N]}\left(e^{2 i k(t-x)}-1\right) g(t, k) d \mu(t) .
$$

To show this, let $I$ be the integral on the right-hand side. We may write this integral in the form

$$
I=\int_{(x, N]} d \mu(t) g(t, k) \int_{x}^{t} d s e^{2 i k(t-s)}=\int_{x}^{N} d s \int_{(s, N]} d \mu(t) g(t, k) e^{2 i k(t-s)} .
$$

This last expression shows that $I$ and hence also the solution $g$ of (5.1) is absolutely continuous and $I^{\prime}=g^{\prime}=-\int_{(x, N]} g(t, k) e^{2 i k(t-x)} d \mu(t)$. Note that it is the right choice to take the interval open at $x$ because this makes $g^{\prime}$ right continuous, as it should be. As for $f=g e^{i k x}$, we thus have that

$$
\begin{gathered}
(A f)(x)=i k e^{i k x}-\int_{[0, N]} \omega(x, t) g(t, k) d \mu(t), \\
\omega(x, t):=\left\{\begin{array}{ll}
0 & 0 \leq t \leq x \\
(1 / 2)\left(e^{i k(2 t-x)}+e^{i k x}\right) & x<t \leq N
\end{array} .\right.
\end{gathered}
$$

Since $\partial \omega / \partial x$ is uniformly bounded, we obtain

$$
(A f)^{\prime}(x)=-k^{2} e^{i k x}-\int_{(x, N]} \frac{\partial \omega}{\partial x} g(t, k) d \mu(t)=-k^{2} f(x, k),
$$

so $f$ solves $-f^{\prime \prime}+\mu f=k^{2} f$, and clearly $f(x)=e^{i k x}$ for $x>N$.

Now given (5.1), we can complete the proof as in [9]. First of all, (5.1) implies that $\|g\|_{\infty} \leq 2$ if $\operatorname{Im} k \geq 0$ and $|k| \geq 2|\mu|([0, N])$. Hence $g(x, k)=1+O\left(|k|^{-1}\right)$ for these $k$. Eq. (5.2) now shows that

$$
\begin{aligned}
(A f)(0) & =i k-\mu(\{0\}) g(0)-\frac{1}{2} \int_{(0, N]}\left(e^{2 i k t}+1\right) g(t) d \mu(t) \\
& =i k-\mu(\{0\})-\frac{1}{2} \int_{(0, N]}\left(e^{2 i k t}+1\right) d \mu(t)+O\left(|k|^{-1}\right) .
\end{aligned}
$$


Similarly,

$$
f(0)=g(0)=1+\frac{1}{2 i k} \int_{(0, N]}\left(e^{2 i k t}-1\right) d \mu(t)+O\left(|k|^{-2}\right) .
$$

The asserted asymptotic formula for $m_{N}=-f(0, k) /(A f)(0, k)$ follows by combining these equations.

The next result gives restrictions on the scalars products on de Branges spaces coming from a generalized Schrödinger equation.

Theorem 5.2. There is an even function $\phi \in B V(-2 N, 2 N)$ with $\phi(0)=-\mu(\{0\})$, such that for every $F \in S_{N}$,

$$
\|F\|_{S_{N}}^{2}=\left\langle f,\left(1+\mathcal{K}_{\phi}\right) f\right\rangle_{L_{2}(0, N)} .
$$

Here $f$ is related to $F$ as in Theorem 4.4.

Note that since $\phi$ is even, it is continuous at $x=0$.

We will not discuss the details of the proof here. One uses the same method as in the proof of [9, Theorem 4.2], with Lemma 5.1 as an important input. So the sought function $\phi$ is defined formally as

$$
\phi(x)=\int \cos \sqrt{\lambda} x d\left(\rho_{N}-\rho_{0}\right)(\lambda),
$$

where $\rho_{N}$ and $\rho_{0}$ are the spectral measures of the half line problems with $\mu_{N} \equiv$ $\chi_{[0, N]} \mu$ and zero potential, respectively. More precisely, the integral over $(-\infty, 0]$ may be evaluated directly (it is a finite sum), and the integral over $(0, \infty)$ is defined as distribution. One then shows by analyzing the Fourier transform with the help of Lemma 5.1b) that this distribution is in fact a function of bounded variation.

As in [9], we have the formula

$$
m_{N}\left(-y^{2}\right)-m_{0}\left(-y^{2}\right)=\frac{1}{y} \int_{0}^{\infty} \phi(t) e^{-y t} d t
$$

which is valid for sufficiently large $y>0$. (The integral makes sense because the method of [9] gives a function $\phi$ defined on all of $\mathbb{R}$.) Fubini's theorem thus shows that

$$
m_{N}\left(-y^{2}\right)-m_{0}\left(-y^{2}\right)=\frac{\phi(0)}{y^{2}}+\frac{1}{y^{2}} \int_{(0, \infty)} e^{-y s} d \phi(s)=\frac{\phi(0)}{y^{2}}+o\left(y^{-2}\right)
$$

as $y \rightarrow \infty$. Comparison with Lemma 5.1 now shows that $\phi(0)=-\mu(\{0\})$, as claimed.

\section{THE INVERSE SPECTRAL THEOREM}

In Theorem 5.2, we associated to each generalized Schrödinger equation a $\phi$ function that determines the scalar product on the corresponding de Branges spaces. Our next goal is to prove the converse: Every function $\phi$ having the properties stated in Theorem 5.2 comes from a generalized Schrödinger equation.

Let

$$
\Phi_{N}:=\left\{\phi \in B V(-2 N, 2 N): \phi \text { even, } 1+\mathcal{K}_{\phi}>0\right\}
$$

be the set of functions that could in principle be a $\phi$ function in the sense of Theorem 5.2. In the last condition, we require that $1+\mathcal{K}_{\phi}$ be positive definite as an operator 
on $L_{2}(0, N)$. This clearly is a necessary condition on $\phi$ if $\left\langle f,\left(1+\mathcal{K}_{\phi}\right) f\right\rangle$ is to define a norm.

Our principal result in this section states that indeed every $\phi \in \Phi_{N}$ occurs as the $\phi$ function (in the sense of Theorem 5.2) of some signed Borel measure $\mu$ on $[0, N)$. We exclude the point $x=N$ here because we have already seen that $\mu(\{N\})$ has no influence on the de Branges space $S_{N}$.

Theorem 6.1. For every $\phi \in \Phi_{N}$, there is a signed Borel measure $\mu$ on $[0, N)$ such that the norm on the de Branges space $S_{N}$ associated with $-d^{2} / d x^{2}+\mu$ on $(0, N)$ is given by

$$
\|F\|_{S_{N}}^{2}=\left\langle f,\left(1+\mathcal{K}_{\phi}\right) f\right\rangle_{L_{2}(0, N)},
$$

where $F(z)=\int f(t) \cos \sqrt{z} t d t$.

The first impression is that the method of [9], suitably extended, should also suffice to prove this result. However, we have not been able to make this approach work. More specifically, we have not succeeded in proving the needed analogs of the smoothness results from [9, Sect. 14]. Rather, we will use an approximation argument that allows us to circumvent these difficulties.

Before proving Theorem 6.1, we note that we have uniqueness in both directions of the correspondence $\mu \leftrightarrow \phi$. This will be used in the proof of Theorem 6.1; of course, it is also of independent interest.

Theorem 6.2. a) If a (finite, signed) Borel measure $\mu$ on $[0, N)$ is given, the $\phi \in \Phi_{N}$ from Theorem 5.2 is unique.

b) If $\phi \in \Phi_{N}$ is given, the $\mu$ from Theorem 6.1 is unique.

This is proved in the same way as the corresponding result (Theorem 5.2) from [9]. Then, in the proof of part b), one now needs the fact that $u(x, z)$ for $x \in[0, N]$, $z \in \mathbb{C}$ determines $\mu$ on $[0, N)$. This indeed holds because $u \mu=u^{\prime \prime}+z u$ and $u(\cdot, z)$ has no zeros for $z \in \mathbb{C} \backslash \mathbb{R}$.

Proof of Theorem 6.1. For absolutely continuous $\phi$, this is proved in [9]. In fact, this is not literally true, because in [9], it is assumed that $\phi(0)=0$, but the extension to general values of $\phi(0)$ (and thus to general boundary conditions) is rather straightforward. We will now approximate the given $\phi \in \Phi_{N}$ by smooth functions $\phi_{n} \in \Phi_{N}$. More precisely, we choose the $\phi_{n} \in C_{0}^{\infty}(-2 N, 2 N)$ so that $\sup _{n}\left\|\phi_{n}^{\prime}\right\|_{L_{1}(-2 N, 2 N)}<\infty$ and $\phi_{n}^{\prime} \rightarrow \phi^{\prime}$ in the $w^{*}$-topology of the space of Borel measures on $(-2 N, 2 N)$. Recall that $\phi^{\prime}$, the distributional derivative of $\phi$, is a measure because $\phi$ itself is of bounded variation. In the sequel, we will also denote this measure by $\nu=\phi^{\prime}$.

To construct a sequence $\phi_{n}^{\prime}$ with the above properties, we can proceed as follows. Fix $\psi \in C_{0}^{\infty}(0,1)$ with $\int \psi=1$. For $n \in \mathbb{N}$ (typically large), subdivide $(-2 N, 2 N)$ into $2 n$ subintervals of length $\ell=2 N / 2 n$. If $I$ is such a subinterval, with endpoints $a$ and $b$, say, define $\phi_{n}^{\prime}$ on $I$ by

$$
\phi_{n}^{\prime}(x)=\frac{\nu(I)}{\ell} \psi\left(\frac{x-a}{\ell}\right) .
$$

Then $\phi_{n}^{\prime} \in C_{0}^{\infty}(-2 N, 2 N)$, these functions are automatically odd, and

$$
\int f(x) \phi_{n}^{\prime}(x) d x \rightarrow \int f(x) d \nu(x)
$$


as $n \rightarrow \infty$ for all $f \in C_{0}(-2 N, 2 N)$; in other words, $\phi_{n}^{\prime} \rightarrow \nu$ in the weak *topology. Moreover, $\int_{I}\left|\phi_{n}^{\prime}\right| \leq|\nu(I)| \int|\psi|$, hence $\left\|\phi_{n}^{\prime}\right\|_{L_{1}(-2 N, 2 N)} \lesssim \sum_{I}|\nu(I)|$. Since $\sum_{I}|\nu(I)| \leq|\nu|((-2 N, 2 N))$, the sequence $\phi_{n}^{\prime}$ is bounded in $L_{1}(-2 N, 2 N)$.

The functions $\phi_{n}$ are still only determined up to a constant. We make the obvious choice, namely, we require that $\phi_{n}(0)=\phi(0)$. Because of the weak $*$-convergence and the norm boundedness in $L_{1}$ of the derivatives, we have that the functions $\phi_{n}(x)$ converge boundedly almost everywhere to $\phi(x)$. More precisely, we will certainly have convergence whenever $\phi(x)=\phi(x-)$, or, equivalently, $\nu(\{x\})=0$.

By dominated convergence this implies that the operators $\mathcal{K}_{\phi_{n}}$ converge to $\mathcal{K}_{\phi}$ in Hilbert-Schmidt norm. In particular, they converge in operator norm and consequently, $1+\mathcal{K}_{\phi_{n}}>0$ for sufficiently large $n$. Hence $\phi_{n} \in \Phi_{N}$ for these $n$.

By [9, Theorem 5.1] (more precisely, by the extension of this result to arbitrary non-Dirichlet boundary conditions), there are potentials $V_{n}$ such that the de Branges spaces associated to $-d^{2} / d x^{2}+V_{n}(x)$ are described by the functions $\phi_{n}$. If we again describe the boundary condition at $x=0$ by $\mu(\{0\})$, then we actually obtain the measures $\mu_{n}=-\phi(0) \delta_{0}+V_{n}$.

More explicitly, the potential $V_{n}$ is given by

$$
V_{n}(x)=\frac{p_{n}^{\prime \prime}(x, x)}{p_{n}(x, x)}
$$

where $p_{n}$ is the solution of the integral equation

$$
p_{n}(x, t)+\int_{0}^{x} K_{\phi_{n}}(t, s) p_{n}(x, s) d s=g_{n}(t)
$$

(see [9, Sect. 18]. In fact, there are two such equations, corresponding to $g_{n} \equiv 1$ and $g_{n}^{\prime \prime}=\phi_{n}, g_{n}(0)=0, g_{n}^{\prime}(0)=1$. With either choice of $g_{n},(6.1)$ holds. Moreover, the zeros of the solutions $p_{n}(x, x)$ form a discrete set, and it does not happen that both solutions have a zero at the same point. So for at least one choice of $p_{n},(6.1)$ is applicable.

We now want to show that $\left(V_{n}\right)$ is bounded in $L_{1}(0, N)$. By the Banach-Alaoglu theorem, this will allow us to extract a subsequence $\left(\mu_{n_{k}}\right)$ that converges to some measure $\mu$ in the $w^{*}$-topology. We will then conclude the proof by showing that $\phi$ is the $\phi$-function of $-d^{2} / d x^{2}+\mu$.

To prove that $\sup _{n}\left\|V_{n}\right\|_{L_{1}(0, N)}<\infty$ it suffices to show that $\sup _{n}\left\|p_{n}^{\prime \prime}\right\|_{L_{1}(0, N)}<$ $\infty$ because of (6.1) and the remarks following (6.2). Denote by $\mathcal{K}_{n}^{(x)}:=\mathcal{K}_{\phi_{n}}^{(x)}$ the integral operator on $L_{2}(0, x)$ generated by the kernel

$$
K_{\phi_{n}}(t, s)=\frac{1}{2}\left(\phi_{n}(t-s)+\phi_{n}(t+s)\right) .
$$

We will consider $\mathcal{K}_{n}^{(x)}$ as an operator on $C[0, x]$ and on $L_{1}(0, x)$. On both of these spaces, we have convergence in operator norm to the corresponding limit operator as $n \rightarrow \infty$. For instance, to compute the $B(C)$ norm of $\mathcal{K}_{n}^{(x)}-\mathcal{K}^{(x)}$, we must analyze expressions of the form

$$
\begin{aligned}
\sup _{\|f\|_{\infty}=1} \sup _{0 \leq t \leq x}\left|\int_{0}^{x}\left(\phi_{n}(t-s)-\phi(t-s)\right) f(s) d s\right| & \\
\leq & \sup _{0 \leq t \leq x} \int_{0}^{x}\left|\phi_{n}(t-s)-\phi(t-s)\right| d s \leq \int_{-x}^{x}\left|\phi_{n}(u)-\phi(u)\right| d u .
\end{aligned}
$$


So we can indeed estimate uniformly in $x \in[0, N]$ :

$$
\left\|\mathcal{K}_{n}^{(x)}-\mathcal{K}^{(x)}\right\| \leq\left\|\phi_{n}-\phi\right\|_{L_{1}(-2 N, 2 N)} .
$$

This estimate also holds for the operator norm in $L_{1}(0, x)$.

Since inversion is a continuous operation, $1+\mathcal{K}_{n}^{(x)}$ is boundedly invertible for sufficiently large $n$ in $C[0, x]$ and in $L_{1}(0, x)$, and the operator norms of the inverses are uniformly bounded in $n$.

Finally, note that $\left\|d^{k} g_{n} / d x^{k}\right\|_{\infty}$ also remains bounded in $n$ for $k=0,1,2$ and either choice of $g_{n}$.

Since $\left(1+\mathcal{K}_{n}^{(x)}\right) p_{n}(x, \cdot)=g_{n}$, we obtain as a first consequence of the above observations that

$$
\left|p_{n}(x, t)\right| \leq C \quad\left(0 \leq t \leq x \leq N, n \geq n_{0}\right) .
$$

Moreover, since $p_{x, n}:=\partial p_{n} / \partial x$ satisfies

$$
p_{x, n}=-p_{n}(x, x)\left(1+\mathcal{K}_{n}^{(x)}\right)^{-1} K_{n}(\cdot, x),
$$

this function is also uniformly bounded. The other first order partial derivative satisfies

$$
p_{t, n}(x, t)=-\int_{0}^{x} K_{t, n}(t, s) p_{n}(x, s) d s+g_{n}^{\prime}(t)
$$

Hence

$$
\sup _{0 \leq t \leq x}\left|p_{t, n}(x, t)\right| \lesssim\left\|\phi_{n}^{\prime}\right\|_{L_{1}(-2 N, 2 N)}+\left\|g_{n}^{\prime}\right\|_{\infty},
$$

and since $\left\|\phi_{n}^{\prime}\right\|_{L_{1}(-2 N, 2 N)}$ also remains bounded, we get a uniform bound on $p_{t, n}$ as well.

The second order derivatives are not necessarily bounded pointwise, so here we work with $L_{1}$ bounds. Apart from this, the reasoning is similar to the arguments used above. By differentiating (6.2) twice with respect to $x$ and solving for $p_{x x, n}$, we obtain

$$
\begin{aligned}
& p_{x x, n}(x, t)= \\
& \quad\left(1+\mathcal{K}_{n}^{(x)}\right)^{-1}\left(-K_{x, n}(t, x) p_{n}(x, x)-\left.K_{n}(t, x)\left(2 p_{x, n}(x, s)+p_{s, n}(x, s)\right)\right|_{s=x}\right) .
\end{aligned}
$$

The expression in parantheses on the right-hand side remains bounded in $L_{1}(0, x)$, uniformly in $x \in[0, N]$, as $n$ varies. Hence $\sup _{n, x}\left\|p_{x x, n}(x, \cdot)\right\|_{L_{1}(0, x)}<\infty$.

Now we are ready to bound $p_{n}^{\prime \prime}(x, x)$. To simplify the notation, we will drop the index $n$ in this final step. By (6.2),

$$
\begin{aligned}
p^{\prime}(x, x) & =\left.\left(p_{x}(x, t)+p_{t}(x, t)\right)\right|_{t=x} \\
& =-K(x, x) p(x, x)-\int_{0}^{x} K(x, s) p_{x}(x, s) d s+g^{\prime}(x)-\int_{0}^{x} K_{x}(x, s) p(x, s) d s .
\end{aligned}
$$

We differentiate one more time. This gives

$$
\begin{gathered}
p^{\prime \prime}(x, x)=-\phi^{\prime}(2 x) p(x, x)-K(x, x) p^{\prime}(x, x)+g^{\prime \prime}(x)-\left.K(x, x) p_{x}(x, s)\right|_{s=x} \\
-2 \int_{0}^{x} K_{x}(x, s) p_{x}(x, s) d s-\int_{0}^{x} K(x, s) p_{x x}(x, s) d s \\
-\left.K_{x}(x, s)\right|_{s=x} \cdot p(x, x)-\int_{0}^{x} K_{x x}(x, s) p(x, s) d s .
\end{gathered}
$$


Our previous results show that everything on the right-hand side except possibly the last term is bounded in $L_{1}$. In the last term, the problem is the occurence of $\phi_{n}^{\prime \prime}$; we do not have control on these functions. However, since $K_{x x}=K_{s s}$, we can integrate by parts:

$$
\begin{aligned}
\int_{0}^{x} K_{x x}(x, s) p(x, s) d s & =\int_{0}^{x} K_{s s}(x, s) p(x, s) d s \\
& =\left.K_{s}(x, s)\right|_{s=x} \cdot p(x, x)-\int_{0}^{x} K_{s}(x, s) p_{s}(x, s) d s
\end{aligned}
$$

So this term is bounded, too. In conclusion, we have that $\sup _{n}\left\|p_{n}^{\prime \prime}(x, x)\right\|_{L_{1}(0, N)}<$ $\infty$, as we wanted to show.

As explained above, we may now assume, by passing to a subsequence if necessary (we will later see that this is actually not necessary), that $V_{n}(x) d x-\phi(0) \delta_{0}$ converges to a (finite, signed) Borel measure $\mu$ on $[0, N]$ in the weak $*$-topology. Since $\sup _{n}\left\|V_{n}\right\|_{L_{1}(0, x)} \rightarrow 0$ as $x \rightarrow 0+$, the limit measure must also satisfy $\mu(\{0\})=$ $-\phi(0)$.

The final step in the proof consists of showing that $\phi$ is the $\phi$ function of $-d^{2} / d x^{2}+\mu$. We will use the following result.

Lemma 6.3. Let $\mu_{n}$ be a bounded sequence of measures (so $\left.\sup \left|\mu_{n}\right|([0, N])<\infty\right)$ that converges to the measure $\mu$ in the weak *-topology. Let $\rho_{n}$ and $\rho$ be the spectral measures of $-d^{2} / d x^{2}+\mu_{n}$ and $-d^{2} / d x^{2}+\mu$, respectively, on $[0, N]$, with boundary conditions $(A f)(0)=0, f^{\prime}(N)=0$. Then $\left(1+\lambda^{2}\right)^{-1} d \rho_{n}(\lambda) \rightarrow\left(1+\lambda^{2}\right)^{-1} d \rho(\lambda)$ in the weak $*$-topology.

Here, the weight $\left(1+\lambda^{2}\right)^{-1}$ is introduced to get finite measures. Assuming Lemma 6.3 , we can complete the proof of Theorem 6.1 as follows. We apply the Lemma with $\mu_{n}=V_{n}(x) d x-\phi(0) \delta_{0}$. For $f \in C_{0}^{\infty}(0, N)$, the transform $F(\lambda)=$ $\int f(x) \cos \sqrt{\lambda} x d x$ goes to zero rapidly as $\lambda \rightarrow \infty$. Moreover, since $\mu_{n}$ is bounded, there is an $E_{0} \in \mathbb{R}$ so that the supports of the $\rho_{n}$ 's and $\rho$ are all contained in $\left(E_{0}, \infty\right)$. Therefore, we can cut off $F(\lambda)$ to the left of $E_{0}$ without changing the integrals $\int|F|^{2} d \rho_{n}, \int|F|^{2} d \rho$. So, $\left(1+\lambda^{2}\right)|F|^{2}$ is an admissable test function and thus

$$
\int_{\mathbb{R}}|F(\lambda)|^{2} d \rho_{n}(\lambda) \rightarrow \int_{\mathbb{R}}|F(\lambda)|^{2} d \rho(\lambda)
$$

On the other hand, $\int|F|^{2} d \rho_{n}=\left\langle f,\left(1+\mathcal{K}_{\phi_{n}}\right) f\right\rangle$ by the identification of $L_{2}\left(\mathbb{R}, d \rho_{n}\right)$ with $S_{N}^{(n)}$ (see Sect. 4). Since

$$
\left\langle f,\left(1+\mathcal{K}_{\phi_{n}}\right) f\right\rangle \rightarrow\left\langle f,\left(1+\mathcal{K}_{\phi}\right) f\right\rangle
$$

and $\int|F|^{2} d \rho=\|F\|_{S_{N}}^{2}$, the uniqueness of $\phi$ (Theorem 6.2b)) together with the fact that $f$ varies over a dense subset of $L_{2}(0, N)$ now show that $\phi$ is the $\phi$ function of $-d^{2} / d x^{2}+\mu$.

It remains to prove Lemma 6.3. We will prove that the corresponding $m$ functions converge, $m_{n}(z) \rightarrow m(z)$, uniformly on compact subsets of $\mathbb{C}^{+}$. As is well known, this implies weak $*$-convergence of the (renormalized) spectral measures (compare $[6])$. The $m$ functions can be computed as follows: $m(z)=-f(0, z) /(A f)(0, z)$, where $f$ solves $-f^{\prime \prime}+\mu f=z f$ and $f^{\prime}(N, z)=0$, and similarly for $m_{n}(z)$. We normalize by further requiring that $f(N, z)=1$ (a multiplicative constant of course 
drops out anyway when $m$ is computed). Then, in analogy to Lemma 4.2 (compare also (5.2)), $f$ is the unique solution of the integral equation

$$
f(x, k)=\cos k(x-N)+\frac{1}{k} \int_{(x, N]} \sin k(t-x) f(t, k) d \mu(t) .
$$

Here, $z=k^{2}$ (since only even functions of $k$ occur, there is no problem with the choice of the square root), and, of course, the $f_{n}$ 's obey the same equations with $\mu$ replaced by $\mu_{n}$. Now we can argue as in the proof of Theorem 2.3. Namely, given a compact subset $K \subset \mathbb{C}$, we pick $\eta>0$ so that

$$
\sup _{0<s \leq \eta} \sup _{z \in K}\left|\frac{\sin k s}{k}\right||\mu|([0, N])<1 .
$$

Then (6.3) shows that $f(x, k)$ remains bounded for $x \in[N-\eta, N]$ and $z \in K$. But now a similar argument may be applied to the interval $[N-2 \eta, N-\eta]$ etc. so that $f$ is eventually seen to be uniformly bounded on $[0, N]$. Clearly, this argument has in fact shown that $f_{n}$ is also bounded, uniformly in $n$. Finally, a slight modification of the whole analysis shows that $f_{n} \rightarrow f$, uniformly in $x \in[0, N]$ and $z \in K$.

Eq. (6.3) implies that

$$
(A f)(0, k)=k \sin k N-\int_{[0, N]} \cos k t f(t, k) d \mu(t) .
$$

Hence we also have that $\left(A f_{n}\right)(0, k) \rightarrow(A f)(0, k)$ uniformly on compact sets. Since it is not possible that $(A f)(0, z)=0$ for non-real $z$, it now follows that $m_{n}(z) \rightarrow$ $m(z)$ locally uniformly, as desired.

\section{REFERENCES}

[1] S. Albeverio, F. Gesztesy, R. Høegh-Kron, and H. Holden, Solvable Models in Quantum Mechanics, Springer-Verlag, Berlin 1988.

[2] J. Brasche, P. Exner, Y. Kuperin, and P. Seba, Schrödinger operators with singular interactions, J. Math. Anal. Appl. 184 (1994), 112-139.

[3] J. Brasche, R. Figari, and A. Teta, Singular Schrödinger operators as limits of point interaction Hamiltonians, Potential Anal. 8 (1998), 163-178.

[4] E.A. Coddington and N. Levinson, Theory of Ordinary Differential Equations, McGraw-Hill, New York 1955.

[5] L. de Branges, Hilbert Spaces of Entire Functions, Prentice-Hall, Englewood Cliffs 1968.

[6] W.F. Donoghue, Monotone Matrix Functions and Analytic Continuation, Springer-Verlag, Berlin 1974.

[7] F. Gesztesy and W. Kirsch, One-dimensional Schrödinger operators with interactions singular on a discrete set, J. Reine Angew. Math. 362 (1985), 28-50.

[8] P. Kurasov and J. Larson, Spectral asymptotics for Schrödinger operators with periodic point interactions, J. Math. Anal. Appl. 266 (2002), 127-148.

[9] C. Remling, Schrödinger operators and de Branges spaces, J. Funct. Anal. 196 (2002), 323394.

[10] J. Weidmann, Spectral theory of ordinary differential operators, Lecture Notes in Mathematics 1258, Springer-Verlag, Berlin 1987.

Universität OsnabrüCK, Fachbereich Mathematik/Informatik, 49069 OsnabrüCK, GerMANY

E-mail address: abamor@mathematik.uni-osnabrueck.de

E-mail address: cremling@mathematik.uni-osnabrueck.de

$U R L:$ www . mathematik. uni-osnabrueck.de/staff/phpages/abamor.rdf.html

$U R L$ : www.mathematik.uni-osnabrueck.de/staff/phpages/remlingc.rdf.html 